\title{
Social touch in mother-infant interaction affects infants' subsequent social engagement and object exploration
}

\author{
Yukari Tanaka $^{1 凶}$, Yasuhiro Kanakogi $^{2} \&$ Masako Myowa (i) ${ }^{3}$
}

Infants' social touch with caregivers has been considered a means of regulating infant physiological and emotional state. In non-human mammals, such regulatory function also facilitates infant exploration and social behavior. However, the types of social touch in human mother-infant interaction that contribute to specific behavioral responses toward people and objects remain unclear. Using a pre- and post-task design, this study investigated the effects of social touch during mother-infant interactions on infants' subsequent preferential looking at social stimuli, social engagement with strangers, and object exploration. Between tasks, mothers and infants spent the time playing in one of two conditions, More Physical Contact or Less Physical Contact. We found that infants in the More Physical Contact Condition showed a greater decrease in evasive behavior with the stranger and enhanced object exploration than those in the Less Physical Contact Condition. Conversely, social touch did not affect infants' preferential looking at static social images. Among the types of social touch, the frequency of affectionate touch reduced evasive behavior to the stranger and facilitated object exploration. These results suggest that social touch, especially affectionate touch, during mother-infant interactions, assist in the modulation of infants' evasive behaviors toward people and object exploration.

\footnotetext{
${ }^{1}$ Department of Social Sciences, Kansai University, Research Fellowships for young scientists, Suita, Japan. ${ }^{2}$ Graduate School of Human Science, Osaka University, Suita, Japan. ${ }^{3}$ Graduate School of Education, Kyoto University, Kyoto, Japan. ${ }^{凶}$ email: yucari.carol@gmail.com
} 


\section{Introduction}

nterpersonal touch modulates social relationships throughout life. Tactile stimulation is a part of the repertoire of mammalian caregiving behaviors (Feldman, 2011), and touching and being touched is crucial for physiological and neurocognitive development in humans and non-human primates (Bremner and Spence, 2017; Cascio et al., 2019; Feldman et al., 2014; Field, 2010). Furthermore, the quality of tactile interactions between caregivers and infants has critical long-term social consequences, affecting the formation of bonds and attachment behaviors throughout the lifespan (Harlow and Zimmermann, 1959; Hertenstein, 2002; Hofer, 1987).

One of the presumed functions of social touch is regulation. Across mammal species, social touch has been shown to decrease stress reactivity. Studies conducted with rodents indicate that the frequency of maternal caregiving behaviors, including licking and grooming, plays an important role in modulating stress reactivity of the infants (Caldji et al., 1998; Kaffman and Meaney, 2007; Liu et al., 1997).

Thus, its function of stress regulation is suggested, as parental touch signals the quality of the environment in which the infant develops, allowing the young to adapt to their environment (Meaney, 2001; Simpson et al., 2019). Tactile stimulation from caregivers across mammalian species triggers a chain of physiological processes of the pups, which leads to decreased stress responses to novel stimulation, and increased their exploration. For example, Simpson and colleagues (2019) carried out a comprehensive study of the effects of early social touch in monkeys. When raised in isolation, tactile stimulation provided by an experimenter decreases the latency with which monkeys approached novel objects and their anxiety when faced with a new experimenter.

Does social touch serve a regulatory function in human adult-infant interactions? Evidence suggests that social touch regulates the emotional and physiological state of infants during social interaction (Feldman et al., 2010; Stack and Muir, 1990, 1992). For example, during a still-face paradigm (Tronick et al., 1978), in which mothers interrupted reciprocal social interaction and posed with a neutral facial expression, infants decrease the time spent gazing and smiling at their mothers and exhibited an increase in negative affect (Adamson and Frick, 2003; Provenzi et al., 2015). When adults continued to touch infants during the still-face period, infants showed a decrease in the overall stress response (i.e., crying) as well as an increase in social attention (i.e., eye contact) and positive affect (i.e., smiles and vocalizations; Stack and Muir, 1990, 1992). Social touch during the still-face period was also shown to regulate infants' physiological state by modulating their parasympathetic nervous system (Feldman et al., 2010). Although there is some evidence that social touch regulates infant internal state, the influence of the experience of social touch on the regulation of infant's subsequent behavior toward the external environment (e.g., objects and other people) that has been reported in non-human mammals is unknown.

In human infant-mother daily interactions, a variety of touch patterns are typically observed, which can be classified into several types (Aso and Iwatate, 2006; Ferber et al., 2008; Jean and Stack, 2009; Weiss et al., 2000). Ferber et al. (2008) described three types of social touch occurring in mother-infant dyad: affectionate touch, including kissing, hugging and holding; stimulating touch, including patting and lifting by the mother, and instrumental touch, which is purposeful utilitarian contact by the mother, including wiping the child's mouth or guiding the child's hand to a toy. Such different types of social touch might regulate infant emotional state during mother-infant interaction, depending on the type of touch. For example, maternal affectionate touch has been demonstrated to be able to notably reduce infants' negative affection (Jahromi et al., 2004). Similarly, gentle stroking touch (classified as affectionate touch) was found to elicit smiling in infants (Jean and Stack, 2009). Stimulating touch (e.g., tickling), on the other hand, increases the arousal of the infant and induces the emotional expression of laughter (Dickson et al., 1997; Srofe and Waters, 1976).

In this study, we investigated how different types of maternal social touch affect infants' subsequent behavioral responses to both people and objects. We utilized three tasks that were performed before and after a mother-infant play interaction. First, the Social Preference task measured infants' preferential looking for static social images, including social/nonsocial scenes and other faces (Telford et al., 2016). The task was aimed at assessing infants' sensitivity to static social images. Mother-infant interaction involving social touch has been found to facilitate infants' attention toward their mothers and their eye contact with their mothers (Elliott et al., 2002; Koester et al., 1989). Thus, social touch would promote infants' preferences for social stimuli (e.g., other people, another's eyes). Second, the Stranger Approach task measured the infants' approaching and evasive behaviors toward a female stranger. Maternal social touch regulates infant negative emotion (Elliott et al., 2002; Jahromi et al., 2004) and facilitates positive emotions (Stack and Arnold, 1998). Thus, the experience of social touch should decrease evasive behavior and facilitate approach behaviors toward other people. Third, the Object Exploration task (Hertenstein and Campos, 2001) was used to evaluate the degree of infants' object exploration. Since social touch promotes subsequent exploratory behavior in non-human primates (Simpson et al., 2019), it can be hypothesized that social touch experiences with their mothers would facilitate human infants' subsequent object exploration.

In the current study, we measured the changes in infants' performance during the three tasks pre-interaction and postinteraction with mothers to control for individual differences in the performance on each task. The pre-task and post-task experimental design was used to measure the immediate effect of maternal behaviors on infants' subsequent behaviors. First, infants participated in the pre-test, which included the three tasks (i.e., social preference, stranger approach, and object exploration). Afterward, mothers and infants played for $\sim 5$ min (i.e., Interaction). During the post-test, infants participated again in each of the three tasks, which were counterbalanced across the infants. During the mother-infant interaction, we created two conditions with varying degrees of social touch called the More Physical Contact Condition (More PC), in which mothers frequently touched infants, and the Less Physical Contact Condition (Less PC), in which mothers touched their infants less frequently to control the level of tactile stimulation experienced by infants. Mother-infant dyads were randomly assigned to one of the two social touch conditions.

We had three main hypotheses. Infants in the More PC Condition would demonstrate (i) a stronger preference for eyes and people, (ii) less evasive behavior and more approaching behavior with the stranger, and (iii) more active exploration of objects compared to those infants assigned to the Less PC Condition. Most importantly, we analyzed the different types of social touch and examined how these forms of social touch influenced infants' task performance.

\section{Method}

Participants. Data from a total of 40 infants aged 6-8 months $\left(M_{\text {age }}=220.08\right.$ days, $\mathrm{SD}=21.74$, range $=184-261$ days; 21 boys, 19 girls) were included in the study. An additional five infants 
( 1 boy) participated in the experiment (two in More PC condition and three in Less PC condition), but their data were excluded for the following reasons: failing to meet the criteria of eye-tracking task (Social Preference Task; $n=2,1$ in More PC condition and one in Less PC condition), breastfeeding between the interaction and post-test $(n=2$, both in Less PC condition), and other technical problems ( $n=1$ in More PC condition). The criteria of the eye-tracking task are described in detail in the "Social preference" task section. All participants were neurologically typical, full-term Japanese infants. The infants' parents gave informed consent, and the study protocol was approved by the Ethics Committee of the Web for Integrated Studies of the Human Mind, Japan (WISH, Japan, No. 26-P-10).

\section{Mother-infant interaction}

Procedure. We observed infants' and mothers' behavior during a mother-infant interaction for $\sim 5 \mathrm{~min}$ in both conditions. After completing the pre-test, mothers and infants sat on the floor of the play space. Mother and infant pairs were assigned to one of two conditions. The More Physical Contact Condition (More PC) involved the mothers playing with the infants while actively touching the infants' body (e.g., holding, tickling, lifting, and singing play). The Less Physical Contact Condition (Less PC) involved the mothers playing while physically separate from the infants (e.g., playing peek-a-boo, watching the infant approach while calling the infants' name, and singing play). Each mother and infant started from the state of face-to-face (sitting or sagittal position), but afterward, their postures were free. The experimenter told mothers to start free play, and after that, the experimenter left to sit behind a partition. Three video cameras (HDR-CX700, HDR-SR1, and HDR-CX535, SONY, Tokyo, Japan) were placed in a position where the infant's face and body, the mother's face and body, and the entire mother and child were reflected, respectively (see the Supplementary Information for further details of the instructions and procedures).

Analysis. The behavior of mothers and infants during the interaction was coded offline using coding software (The Observer XT11, Noldus, Wageningen, The Netherlands). The codes used for the behavior of mothers and infants are shown in Table 1. Since our focus was the amount of social touch during the mother-infant interaction, we made codes of maternal and infant behaviors regarding visual (i.e., gaze and facial expression), auditory (i.e., speech, vocalization, and silence), and tactile (i.e., with or without social touch) modalities, based on previous studies (Ferber et al., 2008; Jahromi et al., 2004; Nishimura et al., 2016; Toda and Fogel, 1993). For coding, we first set the subjects as either mother or infant. Then, whether a behavior occurred was coded every second per subject, and the ratio of the frequency of each behavior was calculated.

The emotional expression of infants was coded as positive or negative emotional expression. Positive emotional expression was defined as the frequency of a smiling facial expression or a laughing facial expression accompanied by laughter. In contrast, negative emotional expression was defined as the frequency of a fussy facial expression or a vocal expression, or a sad facial expression, accompanied by crying. Each code within a modality was mutually exclusive; thus, positive and negative emotional utterances could not co-occur. When the coder could not identify the specific emotional category (e.g., a mixture of positive and negative emotions), the utterance was classified as being a nonspecific vocalization. Among the categories, duplication was allowed (e.g., each individual could show a laughing facial expression accompanied by a laughing voice while seeing the partner's face). Besides the coding that was completed by the main experimenter, another person also coded $20 \%$ of the total data. This coder did not know the purpose of the study but had been trained on how to code the behaviors. We calculated Cohens' kappa $(\kappa)$ for each behavior (all $\kappa$ s $>0.72$; Table 1 ).

After coding the mothers' and infants' behavior, we also coded the types of social touch based on previous studies (Feldman et al., 2004; Ferber et al., 2008; Weiss et al., 2000). The frequencies of seven categories of touch were coded within 30-s timeframes for $5 \mathrm{~min}$ (in the $0-10$ range). The total scores were converted to normal frequencies per minute in the $0-2$ range. The seven categories were included: (1) Holding; (2) Light Active Touch; (3) Passive Touch; (4) Firm Touch; (5) Proprioceptive Stimulation; (6) Vestibular Stimulation; and (7) Matter-of-Fact Touch (Table 2). These seven codes were then combined into three composite scores: Affectionate Touch, Stimulating Touch, and Instrumental Touch. Along with the coding performed by the main experimenter, another person also coded $20 \%$ of the total data with reliability rates between the coders being sufficiently high $(\kappa>0.80)$.

Preliminary analysis of three types of social touch. The average frequency per minute for each type of social touch was: Affectionate Touch $(M=1.21, \mathrm{SD}=0.57$, range: $0-2)$, Stimulating Touch $(M=0.57, \mathrm{SD}=0.43$, range: $0-2)$, and Instrumental Touch $(M=0.14, \mathrm{SD}=0.13$, range: $0-2)$. As preliminary analysis, we compared average frequency of each type of social touch between the two conditions using a two-tailed, independent samples $t$-test. We found significant differences between conditions for the frequency of Affectionate Touch $(M=1.44$, $\mathrm{SE}=0.14$, in the More PC Condition; $M=0.27, \mathrm{SE}=0.09$, in the Less PC condition; $t[38]=6.75, p<0.001, d=2.46)$ and Stimulating Touch $(M=0.81, \mathrm{SE}=0.08$, in the More $\mathrm{PC}$ condition; $M=0.33, \mathrm{SE}=0.07$, in Less PC condition; $t[38]=4.33, p<0.001$, $d=1.35)$. We did not find significant difference between conditions for Instrumental Touch $(M=0.12, \mathrm{SE}=0.02$, in the More PC condition; $M=0.16, \mathrm{SE}=0.03$, in the Less PC condition; $t[38]=-1.08, p=0.28, d=0.31)$.

A hierarchical multiple regression analysis was conducted to examine whether the frequency of each type of social touch affected the infants' performance during each task (Appendix A in Supplementary Information). The dependent variables were calculated by subtracting the frequency of social touch in the pretask and post-test of each task (i.e., preference for a social scene, preference for eyes, approaching behavior and evasive behavior to the stranger, latency to first touch and manipulation duration of an object). In Step 1, the positive emotional expression of the infant during mother-infant interaction served as an independent variable using the Forced Entry method, which was done to confirm that the infant's positive emotional expression did not affect their task performance. In Step 2, the frequencies of Affectionate Touch, Stimulating Touch, and Instrumental Touch were inputted as independent variables using Force Entry to identify what types of social touch affected infants' task performance. When we used Stepwise Selection in Step 2, the results were nearly identical to the Force Entry method.

\section{Details of the pre- and post-tasks Social preference. Stimuli.}

The composition of the stimulus, procedure, and analysis was based on previous research (Telford et al., 2016). We used a Tobii TX300 (Tobii Technology, Stockholm, Sweden) near-infrared gaze tracking system to record eye movement and to present stimuli to infants. The system records near-infrared reflections of both eyes at $60 \mathrm{~Hz}$, while a participant watches an integrated 23inch display. Stimulus presentation and recording were controlled 
Table 1 Coding schema for mother-infant interaction.

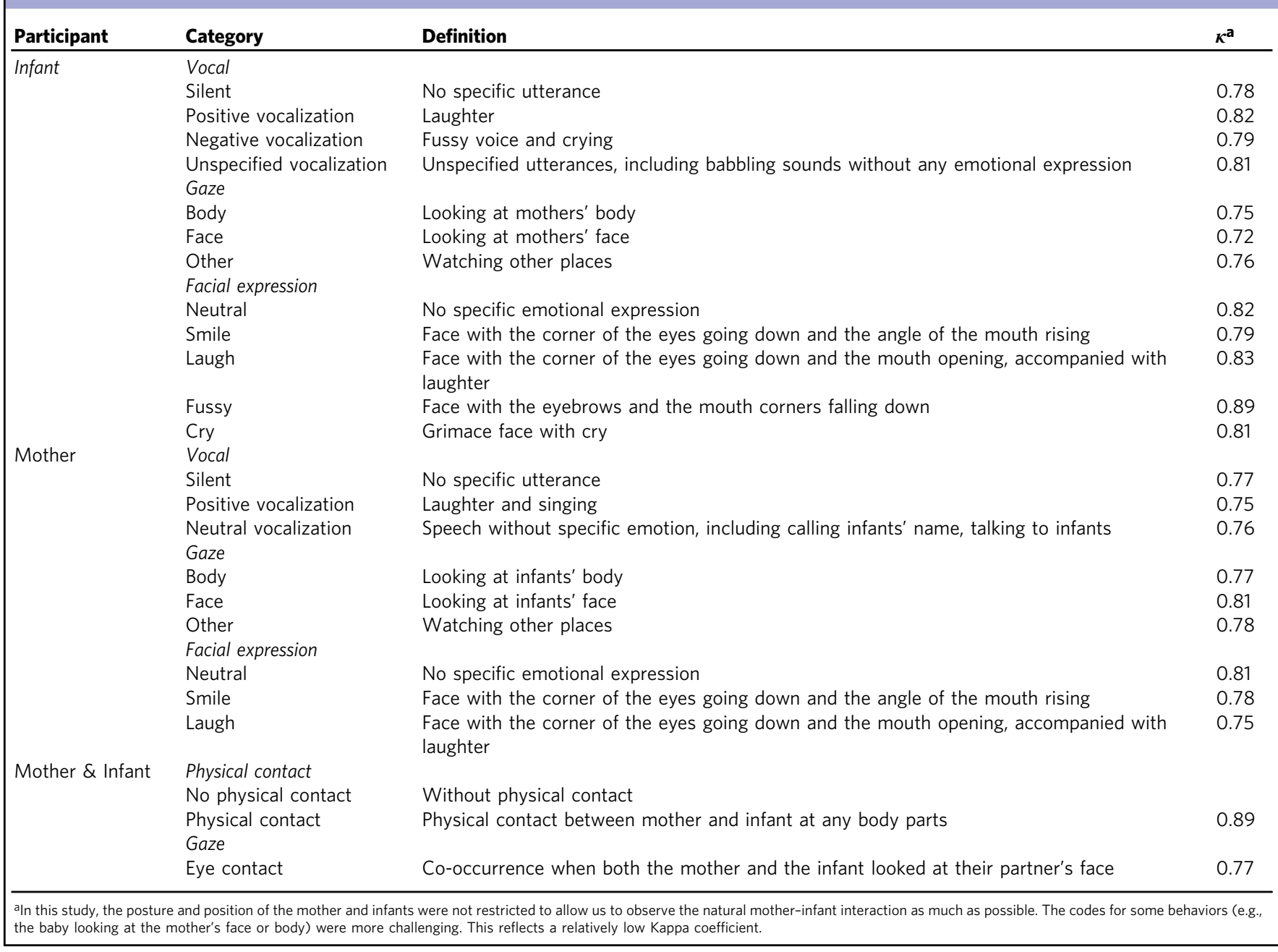

Table 2 Names, definitions, and Kappa coefficients $(\kappa)$ for the three types of touch.

Affectionate touch

Holding

Light active touch

Passive touch

Stimulating touch

Firm touch

Proprioceptive stimulation

Vestibular stimulation

Instrumental touch

Matter-of-fact touch
Affectionately comforting, holding, or hugging in ventral-ventral, ventral-dorsal, or other positions Affectionate kissing, caressing, or stroking

Passive contact, such as resting the hand in contact with the infant

Firm patting, tickling, poking, or massaging with the whole hand

Flexion-extension-flexion of the infant's limbs by the mother

Movements that change the body's orientation in space, including repositioning by lifting, sliding, or rotating the child's body, or swaying or rocking

Purposeful utilitarian contact, such as wiping the child's mouth and guiding the child's hand to a toy via a computer (Dell Precision M6800) using Tobii Studio software (Tobii Technology, Stockholm, Sweden). All stimuli were edited using Adobe Photoshop CS5.6 (Adobe Systems Inc, San Jose, CA). The brightness of all the photos was matched using the same software. Examples of the images used in each task are shown in Supplementary Fig. S1.

The two tests conducted in the current study were as follows: (i) social scenes preferential looking task-there were 16 types of images, with eight images including the face of a child or a baby (i.e., a social scene), and eight images containing only objects (i.e., a nonsocial scene). The size of the images was 1200 pixels in width and 900 pixels in length, and $31.8 \mathrm{~cm}$ in width and $23.8 \mathrm{~cm}$ in height on the center of the screen. The images of the social and the nonsocial scenes were paired and presented side by side. Each image was presented only once to prevent infants from becoming bored. This test consisted of eight stimuli since the two images were combined each time. The combination of images, the left and right positions of the social scene, and the presentation order 
of stimuli were pseudo-randomized, resulting in a total of 16 tests; and (ii) eyes preferential task-the stimuli were four images of adults' faces (two men and two women). The size of the images was 940 pixels in width and 968 pixels in length, and $25.0 \mathrm{~cm}$ in width and $25.6 \mathrm{~cm}$ in height on the center of the screen. Each image was presented only once, and the order of the presentation was randomized.

\section{Procedure.}

Infants and mothers entered a quiet room. Infants were seated on their parent's lap with their eyes $\sim 60-65 \mathrm{~cm}$ from the monitor. The infant's face was monitored through visual observation to check that they paid constant attention to the monitor. Prior to recording, we adjusted the position of participants and the eyetracking monitor to evaluate their stable eye movement, and we performed a five-point calibration procedure before data collection. In this calibration, cartoon animation was presented in the four corners and the center of the display, and the positions of both of the infant's eyes were automatically recorded. After calibration was finished, a series of stimuli were presented. Mothers were instructed to close their eyes during the test to avoid their behavior affecting the infants. Infants watched the same stimuli for both the pre- and post-task.

In the social scenes preferential looking task, each stimulus was presented for $5 \mathrm{~s}$. After four stimuli were presented, cartoon animation was presented as an attention grabber. Once infants attended the attention grabber, the next four stimuli were presented. In the eyes preferential task, each stimulus was presented for $10 \mathrm{~s}$. After two stimuli were presented, cartoon animation was presented as an attention grabber. After infants looked at the attention grabber, the next two stimuli were presented. The total duration of stimulation presentation was $\sim 40 \mathrm{~s}$ in each test. The order of the two tests was counterbalanced across infants. In order to keep the infant's attention on the screen, a short video (about $27 \mathrm{~s}$ ), which was not related to the task, was presented between the two tests.

Analysis. For the social scenes preferential looking task, we set the area of social and nonsocial scenes as Areas of Interest (AOI; Supplementary Fig. S1). In order to investigate the preference for social scenes, we calculated the proportion of fixation duration to social scenes for the whole scene as the Index of Preference for Social Scene (IPS): (social scene)/(social + nonsocial scene) [\%]. For the eyes preferential task, we set the eye and mouth area of the image as AOI (Supplementary Fig. S1). In order to investigate the preference for the eyes, we calculated the percentage of fixation duration to eyes for the sum of eyes and mouth as Index of Preference for Eyes (IPE): (eyes)/(eyes + mouth) [\%]. Data for infants with a total acquisition rate of $<25 \%(n=1)$ shown in the eye-tracking system or infants with a gaze time proportion of $<20 \%$ during stimulus presentation time $(n=1)$ were excluded from further analysis.

\section{Stranger approach. Procedure.}

Procedures of the Stranger Approach Task were primarily based on a previous study (Fox and Davidson, 1987). The stranger was another female experimenter who had no prior knowledge of the infants and who did not interact with the infants and mothers before the experiment. To record the infants' facial expressions, gaze, and behaviors, two video cameras (HDRCX700, and HDR-SR1, SONY, Tokyo, Japan) were placed in the room. One camera was placed in a position where the infant's facial expression and gaze direction were reflected, and the other was placed in the position where the face of the experimenter, mothers, and the infants' bodies were reflected.

The task was conducted in another quiet room, next to the room used for the other tasks. Infants sat on their mother's lap.
The main experimenter made a signal to the stranger, and they appeared at about $1.7 \mathrm{~m}$ from the infant's position. The stranger did not move, maintained a neutral expression, and she looked straight at the infant's face silently. Every $15 \mathrm{~s}$, the stranger approached the infant by $50 \mathrm{~cm}$, with a neutral expression, and looked directly into the infant's face silently. During the last $15 \mathrm{~s}$, the stranger touched the infant's hands and feet with a neutral facial expression. At the end of $60 \mathrm{~s}$, the stranger left from infant's view. The total duration of the task was $60 \mathrm{~s}$. The stranger was the same person for both the pre- and post-tasks.

\section{Analysis.}

We first coded infant behavior for $60 \mathrm{~s}$ using the two video recordings. The coded behaviors included: (i) facial expression (positive, negative, and neutral), (ii) infants' gaze direction (the stranger, mother, and others), (iii) vocal expression (e.g., laughing, fussiness, crying, and other vocalizations), (iv) other behaviors (e.g., reaching for the stranger, touching their own body parts, and pinching their body; Table 3). The behaviors within each area were mutually exclusive, but duplication was allowed between the areas. The behaviors of each area were coded every second by the main experimenter; however, $20 \%$ of the data were coded by another person who did not know the purpose of the present study, and who had been trained on the coding system. The reliability of each code was sufficiently high (Cohens' kappa, «s > 0.77; Table 3).

In order to evaluate the degree of engagement with social interaction, we examined infants' evasive and approach behaviors, including their positive and negative emotional expressions. Approaching behavior was defined as an affiliative behavior shown when approaching strangers, including smiling, neutral vocalization to the stranger, reaching out to the stranger, and laughing in the presence of the stranger. Evasive behavior was defined as fear and avoidance behavior toward others, including fussiness or crying in the presence of the stranger, showing a negative facial expression to the stranger, bending backward to escape from the approach of strangers, and taking away the touched hand. The behaviors between categories were mutually exclusive (i.e., infants never showed Approaching and Evasive Behaviors at the same time), but duplication was allowed within the category. We calculated the percentage of the scores over the $60 \mathrm{~s}$. The mean scores in pre-test for Approaching Behavior were $0.19(\mathrm{SD}=0.23)$ in the More PC coding, and $0.14(\mathrm{SD}=0.19)$ in the Less PC condition. Mean scores for Evasive behavior were $0.16(\mathrm{SD}=0.19)$ in the More PC condition and $0.10(\mathrm{SD}=0.13)$ in the Less PC condition. The dependent variables were the subtracted value of Approaching and Evasive behavior score between the pre- and post-task (post-pre).

\section{Object exploration. Stimuli.}

The stimuli, procedure, and analysis of this task were based on previous research (Putnam and Stifter, 2002; Rothbart, 1988). Two sets of three toys were prepared. One set had low salience (e.g., a tubular building block, a cup, and a triangle building block), and the other set had high salience (e.g., a beeping toy, a screwdriver toy, and a shiny toy). Toy salience means that the toys have visual salience, including color, shape, and motion. There has been some indication that object manipulation is related to emotion regulation in infancy (Hertenstein and Campos, 2001; Putnam and Stifter, 2002; Rothbart, 1988). Since high salient toys give strong visual stimuli, around 8 months of age, infants begin to hesitate to touch these objects (Putnam and Stifter, 2002; Rothbart, 1988). Infants in this study were 6-8-month olds, which is around the age when they begin to hesitate to high salient toys; therefore, we evaluated the object exploratory behavior considering the saliency of the objects. 
Table 3 The coding schema in the stranger approach task.

Behavior

(i) Facial expression

Positive

Negative

Neutral

(ii) Gaze direction

Stranger

Mother

Other

(iii) Vocalization

Neutral utterances

Laughter

Cry

Silent

(iv) Other behaviors

Reaching out

Touch their own body

Plucking their body

Take away the touched hand
Definition

$\kappa$

Facial expression where the corners of the eyes went down and the angle of the mouth rose, or the mouth was open

Facial expression where the eyebrows frowned, the cheeks became tense, and the mouth corners fell down Neutral expression without specific emotion

Looking at the strangers' face or body

Looking at their mothers' face

Seeing infants' own body, mothers' body (i.e., arms or hands), or other area

Utterance, except for laughter or crying

Repeating positive vocalizations accompanied by a smiling face

Repeating negative vocalizations accompanied by a fussy face

0.87

0.78

0.79

No specific vocal behavior

Reaching for the stranger, or touching her.

Touching their own face, limbs, torso or holding their hands in the mouth.
The three toys were placed on a desk, which was $60 \mathrm{~cm}$ in width and $38 \mathrm{~cm}$ in length. When placing the toy set, a blackboard, which was $35 \mathrm{~cm}$ in width and $30 \mathrm{~cm}$ in length, was placed in front of infants so that the toy set would be invisible. There were two cameras in the room to record the infants' behavior. One (Webcam HD720p, Logicool, Lausanne, Switzerland) was placed in front of the infants to focus on their bodies and toys, and the other (HDR535, SONY, Tokyo, Japan) was placed beside them to clearly capture their arms and hands. The high and low salience toys were identical for both the pre- and post-events.

\section{Procedure.}

The task was conducted in a quiet room while the infants sat on their mother's lap. The assistant experimenter put a blackboard in front of infants as a partition. Meanwhile, the main experimenter put the "low salience toys" on the desk. Each toy was placed apart $13 \mathrm{~cm}$ from each other. To prevent mothers from influencing the infants' performance, the examiner told the mothers not to encourage the infants to touch the toys. After checking whether infants paid attention to the board or desk, the assistant removed the blackboard and left the room with the main experimenter. The behavior of the infants for the next $30 \mathrm{~s}$ was recorded by the two cameras. After $30 \mathrm{~s}$, both the experimenter and the assistant entered the room, and the toys were removed. Next, the "high salience toys" were placed in the same positions, and the observation completed identically. The order of presentation order for the toy sets was fixed according to previous studies (Putnam and Stifter, 2002; Rothbart, 1988) since the high salience toys can evoke fearful emotions in infants. If high salient toys were presented first, the elicited fear that occurred could affect the infant's behavior when the low salient toys were presented subsequently. To control such order effect, we first presented low saliency toy.

\section{Analysis.}

To assess the degree of active exploration of objects, the latency to touch a toy (in seconds) and the duration of manipulation were coded from the videotape, based on a previous study (Hertenstein and Campos, 2001). Twenty percent of the total data $(n=8)$ was also analyzed by another person who did not know the purposes of the present study, and the inter-coder reliability was substantially high, with an intraclass correlation coefficient, ICC $(2,1)=0.95$ for latency, and ICC $(2,1)=0.90$ for duration. If infants failed to touch a toy, they were assigned a latency score equal to the duration of the presentation (i.e., $30 \mathrm{~s}$ ), and the duration score was 0 . The pre-test scores for Latency to First Touch were: for low saliency toys, $M=6.95(\mathrm{SD}=5.31)$ in the Less PC condition, and $M=16.10(\mathrm{SD}=11.12)$ in the More PC condition, $t(38)=3.23, p=0.005$; and for high saliency toys, $M=7.98(\mathrm{SD}=5.60)$ in the Less $\mathrm{PC}$ condition, and $M=13.40$ $(\mathrm{SD}=12.44)$ in the More PC condition, $t(38)=1.82, p=0.11$ with Bonferroni correction. The pre-test scores for the Number of Objects were: for the low saliency toys, $M=2.10(\mathrm{SD}=0.64)$ in the Less PC condition, and $M=1.40(\mathrm{SD}=1.19)$ in the More PC condition, $t(38)=2.32, p=0.06$; and for the high saliency toys, $M=2.00(\mathrm{SD}=0.79)$ in the Less $\mathrm{PC}$ condition, and $M=1.45$ $(\mathrm{SD}=1.28), t(38)=1.64, p=0.22$ with Bonferroni correction in the More PC condition. The subtracted values per toy set (low and high salience) between the pre- and post-test (post-pre [second]) were used for the statistical analysis.

\section{Results}

Mothers' and infants' behaviors in the mother-infant interaction. We calculated the mean percentage of the frequency of eye contact, social touch, the mothers' vocalizations, infants' emotional expressions, and mothers' positive expressions, respectively (Table 4). Eye contact was the co-occurrence in the frequency of the mother's attention to the infant's face and the infant's attention to the mother's face. Vocalization of mothers was the mean percentage of the frequency of maternal neutral vocalizations. The behavioral differences between the two conditions were compared using two-tailed, independent samples $t$-tests. We found significant differences between the conditions in terms of the percentage of the frequency of social touch, $t(38)=7.26, p<0.001, d=2.29$, and infant positive emotional expression, $t(38)=3.77, p=0.001, d=1.09$. We found no significant difference between the conditions regarding infants' negative emotional expression, $t(38)=1.28, p=0.21, d=0.41$, maternal vocalization, $t(38)=1.41, p=0.17, d=0.45$, eye contact, $t(38)=1.07, p=0.29, d=0.33$, or maternal positive emotional expression, $t(38)=0.43, p=0.73, d=0.11$. In sum, we confirmed that the amount of maternal social touch was different between the two conditions, but also found that the amount of positive emotional expression in infants also differed between the conditions. 
Table 4 The mean and standard deviation of the duration percentage of maternal and infant behaviors observed in the mother-infant interactions.

\begin{tabular}{|c|c|c|c|c|c|c|}
\hline Condition & $\begin{array}{l}\text { Infant positive expression } \\
M \text { (SD) }\end{array}$ & $\begin{array}{l}\text { Infant negative expression } \\
M \text { (SD) }\end{array}$ & $\begin{array}{l}\text { Social touch } \\
\text { M (SD) }\end{array}$ & $\begin{array}{l}\text { Maternal vocalization } \\
\text { M (SD) }\end{array}$ & $\begin{array}{l}\text { Eye contact } \\
\text { M (SD) }\end{array}$ & $\begin{array}{l}\text { Mother positive } \\
\text { M (SD) }\end{array}$ \\
\hline Less PC & $13.96(7.23)$ & $3.24(7.05)$ & $24.54(34.21)$ & $58.09(8.45)$ & $28.22(16.10)$ & $41.60(22.19)$ \\
\hline
\end{tabular}

Table 5 Mean and standard deviation of index of preference for social scene and index of preference for eyes in the more physical contact and less physical contact conditions.

\begin{tabular}{|c|c|c|c|c|}
\hline \multirow[b]{2}{*}{ Condition } & \multicolumn{2}{|c|}{ Mean IPS [\%] (SD) } & \multicolumn{2}{|c|}{ Mean IPE [\%] (SD) } \\
\hline & $\begin{array}{l}\text { Pre-task } \\
\text { M (SD) }\end{array}$ & $\begin{array}{l}\text { Post-task } \\
\text { M (SD) }\end{array}$ & $\begin{array}{l}\text { Pre-task } \\
\text { M (SD) }\end{array}$ & $\begin{array}{l}\text { Post-task } \\
\text { M (SD) }\end{array}$ \\
\hline More PC & $0.70(0.16)$ & $0.68(0.21)$ & $0.81(0.20)$ & $0.81(0.23)$ \\
\hline Less PC & $0.68(0.17)$ & $0.62(0.19)$ & $0.80(0.19)$ & $0.80(0.30)$ \\
\hline
\end{tabular}

The positive emotional expression of infants could be a covariate of the dependent variables. To examine whether this variable met the criteria for covariates, we conducted a correlation analysis (Pearson's $r$ ) between the positive emotional expression of the infant and the amount of the change (i.e., subtracted values from post- to pre-task) in each task performance (i.e., Object Exploration, Stranger Approach, and Social Preference). Neither the positive emotional expression of infants $(r s-0.10$ to 0.20 , $p s>0.29$, with Bonferroni corrections) nor mothers ( $r s-0.23$ to 0.08 , ps $>0.20$ with Bonferroni corrections), were significantly correlated with the change in the infants' task performances. To eliminate the effect of infant positive emotional expression, we analyzed the dependent variables by using the analysis of covariance (ANCOVA), with infants' positive emotional expression as a covariate.

In addition, to examine aspects of the relationships between maternal social touch and infant positive emotional expression, we conducted correlational analyses between each type of touch (i.e., Affectionate Touch, Stimulating Touch, and Instrumental Touch) and positive emotional expression of infants. We found a significant positive correlation between the frequency of Stimulating Touch and infant positive emotional expression $(r=0.56$, $p=0.004$, with Bonferroni correction), showing that the more frequently mothers engaged in Stimulating Touch (e.g., tickling, poking, or lifting), the more their infants displayed positive emotional expression. On the other hand, we found no significant correlation between Affectionate Touch and infant positive emotional expression ( $r=0.34, p=0.09$, with Bonferroni correction) or between Instrumental Touch and infant positive emotional expression $(r=0.05, \quad p=0.87$, with Bonferroni correction)

\section{Infants' task performance between conditions}

Social preference. Table 5 shows the mean value of the Index of Preference for Social Scene (IPS) and the Index of Preference for Eyes (IPE). We compared IPS with an ANCOVA, with the condition (More PC and Less PC) as a between-participant factor, phase (pre- and post-test) as a within-participant factor, and infants' positive emotional expression as a covariate. We did not find any significant main effects or an interaction ( $F s<2.76, p s>$ $0.11, \eta^{2}<0.07$, in all cases). We also compared IPE with an ANCOVA, with condition as a between-participant factor, phase as a within-participant factor, and infants' positive emotional expression as a covariate. Again, we did not find any significant main effects or an interaction $\left(F s<0.29, p s>0.59, \eta^{2}<0.01\right.$ in all cases). Regarding the types of social touch, we found no significant effects of any type of social touch on social preference (Appendix A in Supplementary Information).

Stranger approach. We compared the mean subtracted percentage of Evasive behavior between the conditions with an ANCOVA, with condition as a between-participant factor, and infants' positive emotional expression as a covariate (see Fig. 1). There was a significant main effect of condition, $F(1,37)=7.42$, $p=0.01, \eta_{\mathrm{p}}^{2}=17$. Infants in the More PC Condition $(M=$ $-0.08, \mathrm{SD}=0.19)$ showed a larger decrease in Evasive behavior compared to those in the Less PC Condition $(M=0.12$, $\mathrm{SD}=0.25)$. We also examined whether Evasive behavior changed after mother-infant interaction with a one-sample, two-tailed $t$-test. We found a significant increase in Evasive Behavior in infants in the Less PC Condition, $t(19)=2.35, p=0.02, d=0.72$, whereas we found only a marginally significant decrease for those in the More PC Condition, $t(19)=-1.87, p=0.08, d=0.58$. In terms of Approaching Behavior, we found no significant main effect of condition, $F(1,37)=0.04, p=0.85, \eta^{2}<0.01$, nor a significant increase from 0 in either condition, $t(19)=0.57$, $p=0.58, d=0.18$ in the Less PC Condition, and $t(19)=1.30$, $p=0.20, d=0.40$ in the More PC Condition.

In addition, we also found a significant relationship between the type of social touch (i.e., Affectionate, Stimulating, and Instrumental Touch) and change in Evasive Behavior using hierarchical multiple regression. Using the subtracted score of Evasive Behavior, the main effect for the frequency of Affectionate Touch was significant at Step $2, \beta=-0.52, t(37)=-2.19$, $p=0.002$, which suggested that there was a negative effect of Affectionate Touch on Evasive Behavior scores. The frequencies of both Stimulating and Instrumental Touch were not related to Evasive Behavior scores. Further, we did not find a significant relationship between any type of touch and Approaching Behavior (Appendix A in Supplementary Information).

Object exploration. The subtracted latency and duration between phases [post-pre] were analyzed using an ANCOVA with condition (More PC and Less PC Conditions) as the betweenparticipant factor, object salience (High Salience and Low Salience) as the within-participant factor and infants' Positive Emotional Expression as the covariates. Regarding latency, we found a significant main effect of condition, $F(1,37)=9.88$, $p=0.003, \eta_{\mathrm{p}}{ }^{2}=0.21$, showing that the decrease in latency was greater for infants in the More PC Condition $(M=-5.83$, $\mathrm{SD}=2.85)$ compared to those in the Less PC Condition $(M=$ $-2.05, \mathrm{SD}=6.07$, Fig. $2 \mathrm{a})$. The negative value means that the 
(a) Evasive behavior

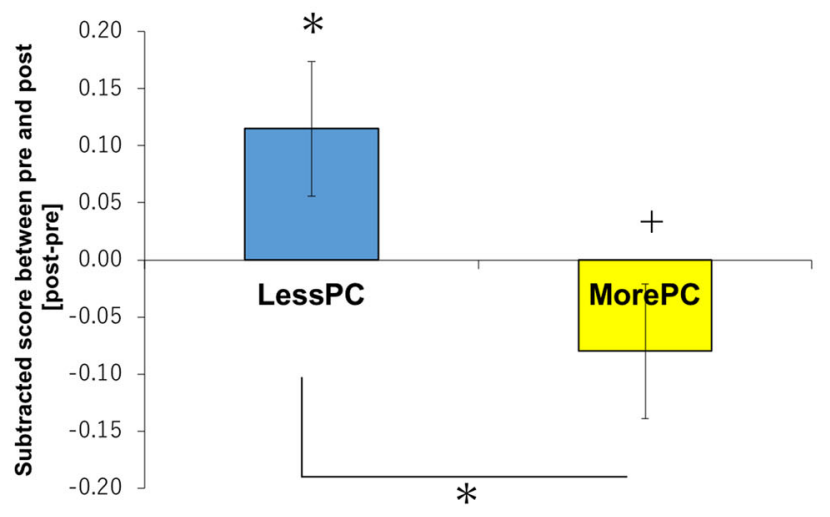

(b) Approaching behavior

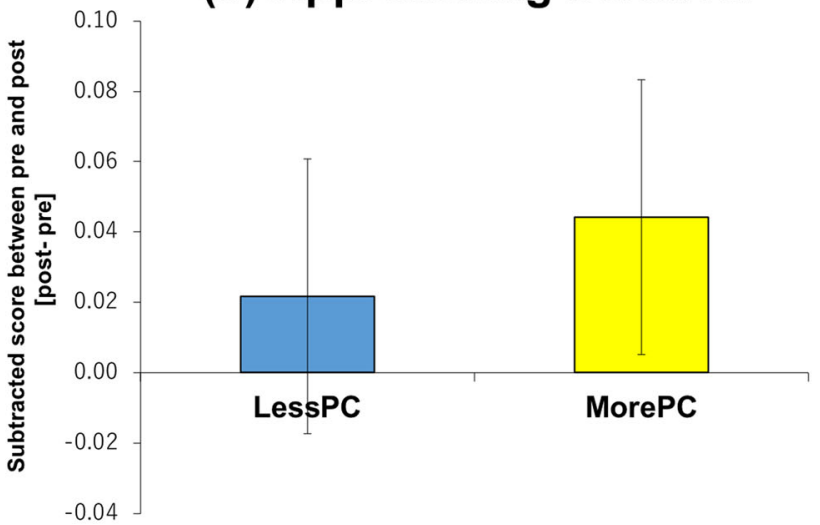

Fig. 1 The results of the Stranger Approach Task. The differential score of infants' reaction to a stranger approach: a Evasive Behavior, $\mathbf{b}$ Approaching Behavior. Error bar shows standard errors. ${ }^{\star} p<0.05,{ }^{+} p<0.10$.

(a) Latency to first touch

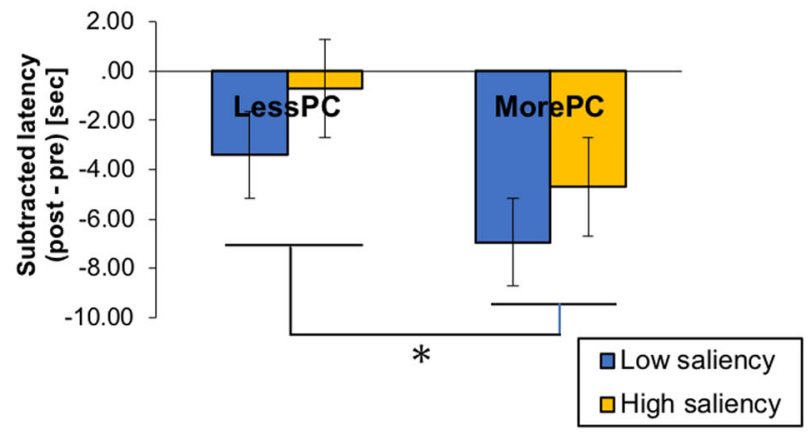

(b) Manipulation duration

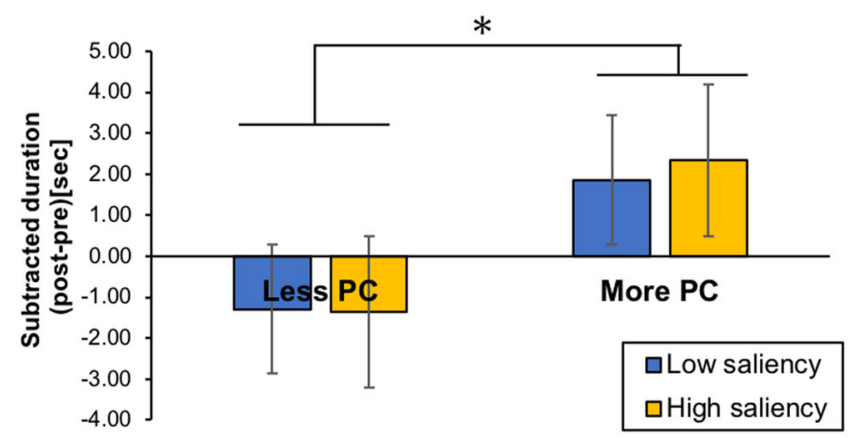

Fig. 2 The results of the Object Exploration Task. Change in a the latency to first touch and $\mathbf{b}$ the duration of object manipulation during the Object Exploration Task; error bars show standard errors. ${ }^{*} p<0.05$.

latency until infants touched objects was significantly shorter in the post-task. We did not find a significant main effect of object type, $F(1,37)=0.21, \quad p=0.65, \quad \eta_{\mathrm{p}}^{2}=0.01$, or interaction between condition and object type, $F(1,37)=0.09, p=0.76$, $\eta_{\mathrm{p}}{ }^{2}=0.002$. Regarding manipulation duration, we also found a significant main effect of condition, $F(1,37)=5.57, p=0.03$, $\eta_{\mathrm{p}}{ }^{2}=0.13$, showing that the increase in duration was greater for infants in the More PC Condition $(M=2.33, \mathrm{SD}=1.09)$ compared to those in the Less PC Condition $(M=-1.55$, $\mathrm{SD}=2.08$; Fig. $2 \mathrm{~b}$ ). We did not find a significant main effect of object type, $F(1,37)=0.65, p=0.43, \eta_{\mathrm{p}}{ }^{2}=0.02$, or an interaction between condition and object type, $F(1,37)=0.02$, $p=0.89, \eta_{\mathrm{p}}^{2}=0.001$.

We also found a significant relationship between the type of social touch (Affectionate, Stimulating, and Instrumental Touch) and object exploration using hierarchical multiple regression. Using the subtracted score of Latency to First Touch, only the frequency of Affectionate Touch significantly affected the Latency to First Touch scores, $\beta=-0.53, t(37)=-3.37, p=0.002$. The frequencies of Stimulating and Instrumental Touch did not significantly predict Latency to First Touch.

The subtracted score of Manipulation Duration was only marginally affected by the frequency of Affectionate Touch, $\beta=0.34, t(37)=2.03, p=0.05$. The frequency of Stimulating and Instrumental Touch were not significantly related to Manipulation Duration (Appendix A in Supplementary Information).

\section{Discussion}

In the present study, we investigated whether and how maternal social touch affected subsequent behavioral responses in infants toward both people and the objects around them. We found that infants in the More PC Condition showed a greater decrease in evasive behaviors toward the stranger, as well as a greater decrease in the latency to their first touch of objects and an increase in the duration of touching objects, compared to infants in the Less PC Condition. Conversely, the frequency of touch did not affect infants' preferential looking at social stimuli, or their approaching behavior toward the stranger. We also found that, among the three types of touch, the frequency of Affectionate Touch significantly predicted the reduction of the Evasive Behavior to the stranger, and the promotion of object exploration. The other two types of touch (i.e., Stimulating Touch and Instrumental Touch) did not affect infants' task performance in any condition. These results suggest that social touch, especially affectionate touch, in mother-infant interaction has the specific role of modulating infants' subsequent evasive behavior to others and their object exploration.

In the Stranger Approach Task, Social Touch was associated with a decrease in Evasive Behaviors toward the stranger (i.e., negative emotional display and refusal to be touched by strangers). In addition, among three types of social touch that were coded, the frequency of Affectionate Touch significantly predicted a reduction in Evasive Behavior. Similarly, previous studies have 
found that social touch (Stack and Muir, 1990, 1992), especially affectionate touch (Jahromi et al., 2004), regulates infants' negative emotions. Therefore, evasive behavior toward the stranger may be suppressed by regulating infants' negative emotions using social touch, especially affectionate touch.

However, we did not find any significant increase in the Approaching Behavior (i.e., positive emotional display, vocalization, and reaching out to the stranger) of infants in our sample. The reason that social touch did not increase approach behavior might be due to the psychological costs of approaching a stranger. In this study, the stranger did not provide infants with any positive social cues, such as a smile or infant-directed speech; thus, her proximity to the infant might have increased infants' negative emotions and evasive behaviors. In fact, we found that Evasive Behavior significantly increased in the Less PC Condition, suggesting that attempting to approach this stranger was more costly than inhibiting evasive behavior.

In the object exploration task, we found that social touch facilitated subsequent object exploration in infants, and of the three types of touch, Affectionate Touch promoted infant object exploration the most. Hertenstein and Campos (2001) examined whether different types of touch affected infants' object exploration. However, their study has found negative consequences of social touch on infants' object exploration. Specifically, tightened touch with high pressure on infants suppressed infants' object exploration when compared to affectionate touch and control touch (i.e., just touching infants' body). In the previous study, infants sat on their mothers' lap. The mothers and infants were not face-to-face; instead, mothers held the infants' abdomens as instructed by the experimenter, which is not a natural form of mother-infant social touch. Thus, unpleasantness might have been enhanced, and negative results were found.

Contrary to our hypothesis, maternal social touch did not affect infants' preferential looking responses toward the static social stimuli. Several potential reasons might explain this. First, infants' attention to the social stimuli might be increased only when perceived at the same time as social touch in a multimodal social interaction. Multimodal interaction refers to the nature of sharing multiple sensory information, including gaze (visual), speech (auditory), and social touch (tactile) during mother-infant interactions. The theorized function of social touch in multimodal interactions is to enhance infants' attention and facilitate their learning. Seeing others' faces or hearing others' voices simultaneously with social touch attracts the infants' attention to faces or voices, which facilitates their learning (Della Longa et al., 2019; Seidl et al., 2015; Tanaka et al., 2018). However, in this study, the social stimuli in the task were spatiotemporally separated from the experience of social touch during the mother-infant interaction, so the presentation of social stimulation and the experience of social touch were not simultaneous. Thus, infants' attention might not have affected by social touch.

Another reason might be the physical properties of task stimuli. We prepared stimuli that were consistent with previous studies. While the brightness of the photos was matched between the photos, we might not have had complete control over the photo complexity, such as the elements and symmetry of the stimuli. These subtle differences could have affected the results. Future research should control for this and examine any differences in infants' behavior.

Why might maternal touch, especially affectionate touch, modulate evasive behavior toward strangers and promote infants' object exploration? The effects of social touch observed in this experiment can be interpreted as a regulatory function of the social contact early in life that is common among nonhuman mammals, including mouse (Meaney, 2001), and monkeys (Simpson et al., 2019). For example, social touch relieves stress at the pups' physiological level and facilitates their exploration (Meaney, 2001; Simpson et al., 2019). Its underlying psychological mechanism could be explained by the BIS/BAS Model, which describes human behavior through two motivational systems-the Behavioral Inhibition System (BIS) and the Behavioral Activation System (BAS) (Gray, $1970,1981,1982,1987)$. BIS is a motivational system that is activated by punishment signals or the absence of reward signals that cause frustration. This system calls attention to potential threat stimuli and as a result, individuals suppress their behavior. On the other hand, BAS is a motivational system that is activated by conditioned stimuli that signal a reward or the absence of punishment, which drives ones' behaviors toward achieving goals. In the present study, social touch may have functioned as a conditioned stimulus for the signal to activate the BAS system. During social interactions, affectionate touch is a positive experience that provides comfort to infants (Peláez-Nogueras et al., 1997). The accumulation of such experiences indicates that social touch, especially the affectionate touch, functions as a conditioned stimulus to inform infants of a reward or the absence of punishment. Thus, they might have increased infants' motivation to enhance object exploration and reduce evasive behavior toward the stranger.

Likewise, social touch during mother-infant interactions, particularly affectionate touch, could regulate infants' physiological state. This regulation may be explained by C-Tactile fibers (CT-fibers), which are peripheral nerve fibers that are activated during gentle touch at a slow speed (McGlone et al., 2014). Activation of CT-fibers accelerates the parasympathetic nervous system and regulates stress reactivity. Infants' heart rates have been shown to be the most stable when being touched at a slow stroking speed $(2-3 \mathrm{~cm} / \mathrm{s}$; Aguirre et al., 2019; Fairhurst et al., 2014), suggesting that the activation of CT-fibers relieves the infants' physiological stress. Such physiological changes in infants can also be related to changes in their subsequent behavior toward objects and other people.

There are several limitations to this study. First, factors that contribute to infants' behaviors other than maternal social touch could not be eliminated entirely. We also found significant differences in the positive emotional displays of infants between the three conditions as well as the amount of social touch, during mother-infant interaction. To control the positive emotional expression of infants, an analysis of covariates (ANCOVAs) and hierarchical multiple regression analysis were conducted, wherein we controlled for infants' positive emotional expression. Results showed no significant effects of positive emotional expression on any task performance of the infants. In addition, the frequency of infants' positive emotional expression was positively correlated with maternal stimulating touch, suggesting that maternal stimulating touch during mother-infant interactions induced the infant's immediate positive emotional expression. Therefore, it is unlikely that infants' positive emotions affected the infants' behavior during subsequent tasks.

Second, since the present study examined the effect of social touch in naturally occurring mother-infant interaction, affectionate touch and stimulating touch sometimes co-occurred (e.g., a mother tickled the infant's body while holding the baby). Although affectionate touch significantly predicted the change of infant evasive behavior to the stranger and object exploration, only the effect of affectionate touch could not be perfectly extracted from the interaction.

The third limitation is that we were unable to examine infants' arousal during mother-to-child interactions. In this study, we analyzed the infants' emotions during mother-infant interactions and confirmed that their emotions did not affect the task. However, infants' arousal level might have been correlated with emotion. In the future, research should simultaneously evaluate the arousal of infants during these tasks. 
A fourth limitation is that manipulation of the amount of physical contact in our experimental design may have distorted the natural form of play in the mother-infant interaction. Some behaviors could have had an "anomaly effect" (for instance, tickling without touching), which could violate infants' expectation and trigger negative emotions. As a result, the infants may not have performed as well as they could have in the test phase. For future research, we should use an experimental design to examine mother-infant interactions to eliminate such anomaly effects.

In summary, maternal social touch during mother-infant interactions was shown to affect infants' subsequent behaviors toward both people and objects around them. Infants who engaged in more frequent contact with their mother showed a greater decrease in evasive behaviors toward a stranger as well as greater facilitation of object exploration compared to infants receiving less physical context. Among the three types of social touch, the frequency of affectionate touch was found to predict the reduction of infants' evasive behavior toward the stranger, and the promotion of object exploration. These findings suggest that, of the different types of social touch in mother-infant interactions, affectionate touch has a particularly critical role of modulating infants' behaviors and attitudes toward both people and objects in their environment.

\section{Data availability}

The datasets generated during and/or analyzed during the current study are available from the corresponding author on reasonable request.

Received: 3 December 2019; Accepted: 29 September 2020; Published online: 01 February 2021

\section{References}

Adamson LB, Frick JE (2003) The still face: a history of a shared experimental paradigm. Infancy 4:451-473. https://doi.org/10.1207/S15327078IN0404_01

Aguirre M, Couderc A, Epinat-Duclos J, Mascaro O (2019) Infants discriminate the source of social touch at stroking speeds eliciting maximal firing rates in CT-fibers. Dev Cogn Neurosci 36:100639. https://doi.org/10.1016/j. dcn.2019.100639

Aso N, Iwatate S (2006) Differences in mothers' touching their infants who were $0-1$ year-old among the seven scenes of nurturing: using the retrospective method. J Child Health 65:488-497

Bremner AJ, Spence C (2017) The development of tactile perception. Adv Child Dev Behav 52:227-268. https://doi.org/10.1016/bs.acdb.2016.12.002

Caldji C, Tannenbaum B, Sharma S, Francis D, Plotsky PM, Meaney MJ (1998) Maternal care during infancy regulates the development of neural systems mediating the expression of fearfulness in the rat. Proc Natl Acad Sci USA 95:5335-5340. https://doi.org/10.1073/pnas.95.9.5335

Cascio CJ, Moore D, McGlone F (2019) Social touch and human development. Dev Cogn Neurosci 35:5-11. https://doi.org/10.1016/j.dcn.2018.04.009

Della Longa L, Gliga T, Farroni T (2019) Tune to touch: affective touch enhances learning of face identity in 4-month-old infants. Dev Cogn Neurosci 35:42-46. https://doi.org/10.1016/j.dcn.2017.11.002

Dickson K, Walker H, Fogel A (1997) The relationship between smile-type and play-type during parent-infant play. Dev Psychol 33:925-933. https://doi.org/ 10.1037/0012-1649.33.6.925

Elliott MR, Reilly SM, Drummond J, Letourneau N (2002) The effect of different soothing interventions on infant crying and on parent-infant interaction. Infant Ment Health J 23:310-328. https://doi.org/10.1002/imhj.10018

Fairhurst MT, Löken L, Grossmann T (2014) Physiological and behavioral responses reveal 9-month-old infants' sensitivity to pleasant touch. Psychol Sci 25:1124-1131. https://doi.org/10.1177/0956797614527114

Feldman R (2011) Maternal touch and the developing infant. In: Hertenstein MJ, Weiss SJ (eds) The handbook of touch: neuroscience, behavioral, and health perspectives. Springer, New York, pp. 373-407

Feldman R, Keren M, Gross-Rozval O, Tyano S (2004) Mother-child touch patterns in infant feeding disorders: relation to maternal, child, and environmental factors. J Am Acad Child Adolesc Psychiatry 43:1089-1097. https:// doi.org/10.1097/01.chi.0000132810.98922.83
Feldman R, Rosenthal Z, Eidelman AI (2014) Maternal-preterm skin-to-skin contact enhances child physiologic organization and cognitive control across the first 10 years of life. Biol Psychiatry 75:56-64. https://doi.org/10.1016/j. biopsych.2013.08.012

Feldman R, Singer M, Zagoory O (2010) Touch attenuates infants' physiological reactivity to stress. Dev Sci 13:271-278. https://doi.org/10.1111/j.14677687.2009.00890.x

Ferber SG, Feldman R, Makhoul IR (2008) The development of maternal touch across the first year of life. Early Hum Dev 84:363-370. https://doi.org/ 10.1016/j.earlhumdev.2007.09.019

Field T (2010) Touch for socioemotional and physical well-being: a review. Dev Rev 30:367-383. https://doi.org/10.1016/j.dr.2011.01.001

Fox NA, Davidson RJ (1987) Electroencephalogram asymmetry in response to the approach of a stranger and maternal separation in 10-month-old infants. Dev Psychol 23:233-240. https://doi.org/10.1037/0012-1649.23.2.233

Gray JA (1970) The psychophysiological basis of introversion-extraversion. Behav Res Ther 18:249-266. https://doi.org/10.1016/0005-7967(70)90069-0

Gray JA (1981) A critique of Eysenck's theory of personality. In: Eysenck HJ (ed) A model for personality. Springer, Berlin, pp. 246-276.

Gray JA (1982) The neuropsychology of anxiety. Oxford University Press, Oxford

Gray JA (1987) The psychology of fear and stress, 2nd edn. Oxford University Press, Oxford

Harlow HF, Zimmermann RR (1959) Affectional responses in the infant monkey: orphaned baby monkeys develop a strong and persistent attachment to inanimate surrogate mothers. Science 130:421-432. https://doi.org/10.1126/ science.130.3373.421

Hertenstein MJ (2002) Touch: its communicative functions in infancy. Hum Dev 45:70-94. https://doi.org/10.1207/S15327078IN0204_09

Hertenstein MJ, Campos JJ (2001) Emotion regulation via maternal touch. Infancy 2:549-566. https://doi.org/10.1207/S15327078IN0204_09

Hofer MA (1987) Early social relationships: a psychobiologist's view. Child Dev 58:633-647. https://doi.org/10.2307/1130203

Jahromi LB, Putnam SP, Stifter CA (2004) Maternal regulation of infant reactivity from 2 to 6 months. Dev Psychol 40:477-487. https://doi.org/10.1037/00121649.40.4.477

Jean ADL, Stack DM (2009) Functions of maternal touch and infants' affect during face-to-face interactions: new directions for the still-face. Infant Behav Dev 32:123-128. https://doi.org/10.1016/j.infbeh.2008.09.008

Kaffman A, Meaney MJ (2007) Neurodevelopmental sequelae of postnatal maternal care in rodents: clinical and research implications of molecular insights. J Child Psychol Psychiatry 48:224-244. https://doi.org/10.1111/j.1469-7610.2007.01730.x

Koester LS, Papousek H, Papousek M (1989) Patterns of rhythmic stimulation by mothers with three-month-olds: a cross-modal comparison. Int J Behav Dev 12:143-154. https://doi.org/10.1177/016502548901200201

Liu D, Diorio J, Tannenbaum B et al. (1997) Maternal care, hippocampal glucocorticoid receptors, and hypothalamic-pituitary-adrenal responses to stress. Science 277:1659-1662. https://doi.org/10.1126/science.277.5332.1659

McGlone F, Wessberg J, Olausson H (2014) Discriminative and affective touch: sensing and feeling. Neuron 82:737-755. https://doi.org/10.1016/j. neuron.2014.05.001

Meaney M (2001) Maternal care, gene expression, and the transmission of individual differences in stress reactivity across generations. Annu Rev Neurosci 24:1161-1192. https://doi.org/10.1146/annurev.neuro.24.1.1161

Nishimura Y, Kanakogi Y, Myowa-Yamakoshi M (2016) Infants' emotional states influence maternal behaviors during holding. Infant Behav Dev 43:66-74. https://doi.org/10.1016/j.infbeh.2016.02.003

Peláez-Nogueras M, Field T, Gewirtz JL, Cigales M, Gonzalez A, Sanchez A, Richardson SC (1997) The effects of systematic stroking versus tickling and poking on infant behavior. J Appl Dev Psychol 18:169-178. https://doi.org/ 10.1016/S0193-3973(97)90034-4

Provenzi L, Borgatti R, Menozzi G, Montirosso R (2015) A dynamic system analysis of dyadic flexibility and stability across the face-to-face still-face procedure: application of the state space grid. Infant Behav Dev 38:1-10. https:// doi.org/10.1016/j.infbeh.2014.10.001

Putnam SP, Stifter CA (2002) Development of approach and inhibition in the first year: parallel findings from motor behavior, temperament ratings and directional cardiac response. Dev Sci 5:441-451. https://doi.org/10.1111/1467-7687.00239

Rothbart MK (1988) Temperament and the development of the inhibited approach. Child Dev 59:1241-1250. https://doi.org/10.2307/1130487

Seidl A, Tincoff R, Baker C (2015) Why the body comes first: effects of experimenter touch on infants' word finding. Dev Sci 18:155-164. https://doi.org/ 10.1111/desc. 12182

Simpson EA, Sclafani V, Paukner A, Kaburu SSK, Suomi SJ, Ferrari PF (2019) Handling newborn monkeys alters later exploratory, cognitive, and social behaviors. Dev Cogn Neurosci 35:12-19. https://doi.org/10.1016/j.dcn.2017.07.010

Srofe LA, Waters E (1976) The ontogenesis of smiling and laughter: a perspective on the organization of development in infancy. Psychol Rev 83:173-189. https://doi.org/10.1037/0033-295X.83.3.173 
Stack DM, Arnold SL (1998) Changes in mothers' touch and hand gestures influence infant behavior during face-to-face interchanges. Infant Behav Dev 21:451-468. https://doi.org/10.1016/S0163-6383(98)90019-4

Stack DM, Muir DW (1990) Tactile stimulation as a component of social interchange: new interpretations for the still-face effect. $\mathrm{Br} \mathrm{J}$ Dev Psychol 8:131-145. https://doi.org/10.1111/j.2044-835X.1990.tb00828.x

Stack DM, Muir DW (1992) Adult tactile stimulation during face-to-face interactions modulates five-month-olds' affect and attention. Child Dev 63:1509-1525. https://doi.org/10.2307/1131572

Tanaka Y, Kanakogi Y, Kawasaki M, Myowa M (2018) The integration of audiotactile information is modulated by multimodal social interaction with physical contact in infancy. Dev Cogn Neurosci 30:31-40. https://doi.org/ 10.1016/j.dcn.2017.12.001

Telford EJ, Fletcher-Watson S, Gillespie-Smith K et al. (2016) Preterm birth is associated with atypical social orienting in infancy detected using eye tracking. J Child Psychol Psychiatry 57:861-868. https://doi.org/10.1111/jcpp.12546

Toda S, Fogel A (1993) Infant response to the still-face situation at 3 and 6 months. Dev Psychol 29:532-538. https://doi.org/10.1037/0012-1649.29.3.532

Tronick E, Als H, Adamson L, Wise S, Brazelton TB (1978) The infant's response to entrapment between contradictory messages in face-to-face interaction. J Am Acad Child Psychiatry 17:1-13. https://doi.org/10.1016/S0002-7138(09)62273-1

Weiss SJ, Wilson P, Hertenstein MJ, Campos R (2000) The tactile context of a mother's caregiving: implications for attachment of low birth weight infants. Infant Behav Dev 23:91-111. https://doi.org/10.1016/S0163-6383(00)00030-8

\section{Acknowledgements}

This study was supported by funding from the Grants-in-Aid for Scientific Research from the Japan Society for the Promotion of Science and the Ministry of Education Culture, Sports, Science and Technology (24119005, 24300103 to M.M.), the Center of Innovation Program from Japan Science and Technology Agency, JST to M.M., and the Mayekawa Foundation to M.M. (2015-2016), and Y.T. (2015-2016). We would like to thank all the children and parents who participated in the research. We also thank K. Kobayashi, M. Yamamoto, and M. Yoshii for their assistance in the experiment.

\section{Author contributions}

Y.T., Y.K., and M.M. designed the research. Y.T. conducted experiments and analyzed the data. Y.T., Y.K., and M.M. wrote the main manuscript text.

\section{Competing interests}

The authors declare no competing interests.

\section{Additional information}

Supplementary information is available for this paper at https://doi.org/10.1057/s41599 020-00642-4.

Correspondence and requests for materials should be addressed to Y.T.

Reprints and permission information is available at http://www.nature.com/reprints

Publisher's note Springer Nature remains neutral with regard to jurisdictional claims in published maps and institutional affiliations.

\section{(c) (i)}

Open Access This article is licensed under a Creative Commons Attribution 4.0 International License, which permits use, sharing, adaptation, distribution and reproduction in any medium or format, as long as you give appropriate credit to the original author(s) and the source, provide a link to the Creative Commons license, and indicate if changes were made. The images or other third party material in this article are included in the article's Creative Commons license, unless indicated otherwise in a credit line to the material. If material is not included in the article's Creative Commons license and your intended use is not permitted by statutory regulation or exceeds the permitted use, you will need to obtain permission directly from the copyright holder. To view a copy of this license, visit http://creativecommons.org/ licenses/by/4.0/.

(C) The Author(s) 2021 